\title{
Contribution of the Medical Imaging in Care of Ambiguous Genitalia
}

\section{Zanga Sore Moussa ${ }^{1}$, Diallo Ousseini ${ }^{2}$, Ouattara Boubakar ${ }^{2}$, Napon Aischa Madina ${ }^{1}$, Bamouni Yomboue Abel ${ }^{2}$, Cisse Rabiou²}

\author{
${ }^{1}$ Radiology Department of Charles de Gaulle Teaching Paediatric Hospital, Ouagadougou, Burkina Faso \\ ${ }^{2}$ Radiology Department of Yalgado Ouédraogo Teaching Hospital, Ouagadougou, Burkina Faso \\ Email: *zasomo@yahoo.fr
}

How to cite this paper: Moussa, Z.S., Ousseini, D., Boubakar, O., Madina, N.A., Abel, B.Y. and Rabiou, C. (2018) Contribution of the Medical Imaging in Care of Ambiguous Genitalia. Open Journal of Radiology, 8, 125-130.

https://doi.org/10.4236/ojrad.2018.82014

Received: May 4, 2018

Accepted: June 25, 2018

Published: June 28, 2018

Copyright $\odot 2018$ by authors and Scientific Research Publishing Inc. This work is licensed under the Creative Commons Attribution International License (CC BY 4.0).

http://creativecommons.org/licenses/by/4.0/

\begin{abstract}
We report a case of ambiguous genitalia in a 15-year-old patient to demonstrate input of medical imaging in the diagnosis and care of this rare congenital deformation. Disorders of sexual differentiation (DSD) have been defined as congenital conditions in which development of chromosome, gonad, or anatomic sex is atypical and that raise an issue in the sex assignment. In lower socioeconomic countries where antenatal diagnosis is almost non-existent, due to lack of technical facilities, their medical and surgical management is hard. The case which we report was investigated by ultrasonography and genitography, which remains primary indications in our context.
\end{abstract}

\section{Keywords}

Ambiguous Genitalia, Karyotype, Ultrasonography, Genitography

\section{Introduction}

Ambiguous genitalia or hermaphroditism is a rare intersex condition. It affects about one newborn in 4500 births and is defined by mixed various proportions of male and female sexual characteristics [1]. According to the new nomenclature, we no longer speak of ambiguous genitalia but of disorders of sexual differentiation (DSD). This classification is based on the karyotype [2]. DSD's abnormalities result from insufficient masculinization of a genetically male embryo (46 XY, DSD) or excessive virilization of a female embryo (46 XX, DSD), in which several genetic and hormonal factors are involved [3].

Radiological imaging plays an important role in demonstrating genital anatomy [4]. We report a case of true hermaphroditism in a 15-year-old girl to illustrate the role of radiological imaging in diagnosis of this rare condition, as well 
as issue of its management in our context.

\section{Observation}

A 15-year-old patient, with history of scrotal hernia repaired at 4 months age, was referred to the radiology department of Yalgado Ouedraogo Teaching Hospital for imaging further investigation of an abnormal of sexual differentiation. Clinically, there was regular menses per month for about 3 years. Physical examination revealed male and female external genitalia (Figure 1). However, there was no vulva visible at the perineum and hypospadias was noted. Radiological evaluation performed consisted of an abdominal and pelvic ultrasound scan, scrotal ultrasound scan and genitography. Pelvic ultrasound scan showed a $\sim 3.7 \mathrm{~cm}$ length retro-vesical tubular structure likely a rudimentary uterus. In the right ovarian lodge, there was a $\sim 14 \times 8 \mathrm{~mm}$ ovarian tissue with normal stoma and follicles. The left ovary was not demonstrated (Figure 2).

The bladder has a normal outline. No calculus or mass noted. There was no evidence of retrovesical prostatic tissue. The liver, bile ducts, pancreas, spleen, and both kidneys showed normal size and echopattern. Scrotal ultrasound scan demonstrated a single testicle with normal epididymal cap and measured $44 \mathrm{~mm}$ $\times 28 \mathrm{~mm} \times 25 \mathrm{~mm}$ (Figure 3). Its echopattern was heterogeneous due to presence of calcified, cystic and fibrous areas reflecting a fairly severe dysplasia. Scrotal layers were not thickened and there was no hydrocele. Corpora cavernosa appeared homogeneous, without any recent or fibrous lesions. Genitogram was performed to better evaluate the first sonographic findings on the internal genitalia. After grooming, disinfection of the meatus (hypospadias) and perineum, we catheterized urethra till bladder with a No.8 rigid catheter bringing back urine (Figure 4). We set up another rectal balloon catheter. Contrast medium flowed freely, bladder and rectum were outlined. There were a significant inter vesico-rectal space indicating presence of a uterus.

A third rigid catheter was inserted in the vagina and after several attempts, we were able to outline uterus with contrast medium. It appeared tubular and small. Struggling, we demonstrated a large, straight right fallopian tube but there were no ampullary folds or intraperitoneal spillage (Figure 5(a) and Figure 5(b)). This imaging assessment confirmed the diagnosis of true hermaphroditism. Even before the result of karyotype tests, which was $46 \mathrm{XX}$, the patient and his parents had chosen the option of keeping male characters. The patient underwent bilateral mastectomy (Figure 5) first and then hysterectomy as well as right salpingectomy. Postoperative recoveries were uneventful and good psychological tolerance was observed both in patient and his close contacts.

\section{Discussion}

DDS are rare diseases with a prevalence of $0.1 \%$ to $2 \%$ according to some authors and $2.3 \%$ in a recent study conducted in Mali [3] [4]. They lead a discordance between the sex itself ("phenotypic") and the genetic sex ("genotype"). 


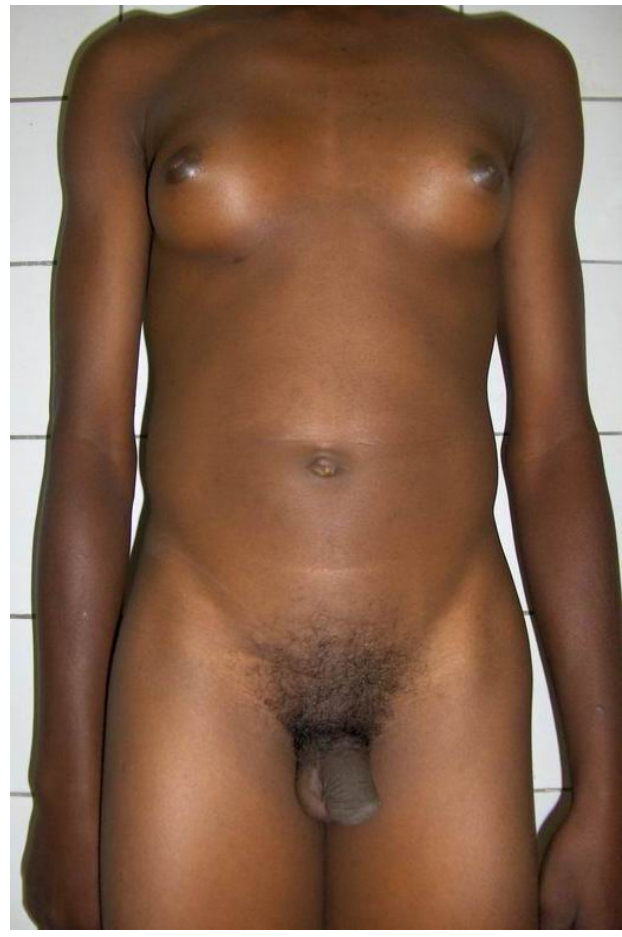

Figure 1. Photographic picture showing external genitalia of our patient.
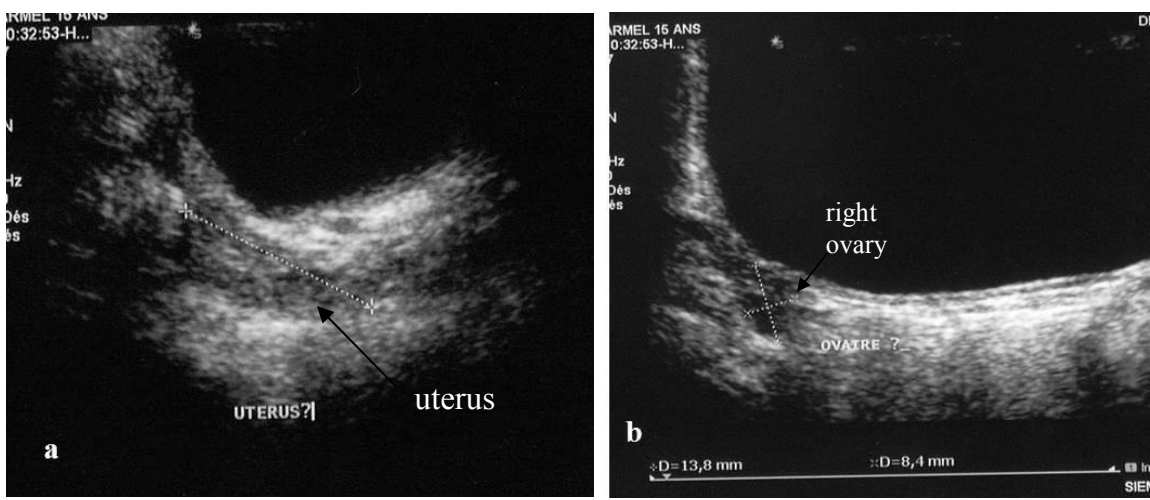

Figure 2. Pelvic ultrasound in sagittal view (a) showing a small uterus and in axial view; (b) showing the ovary.

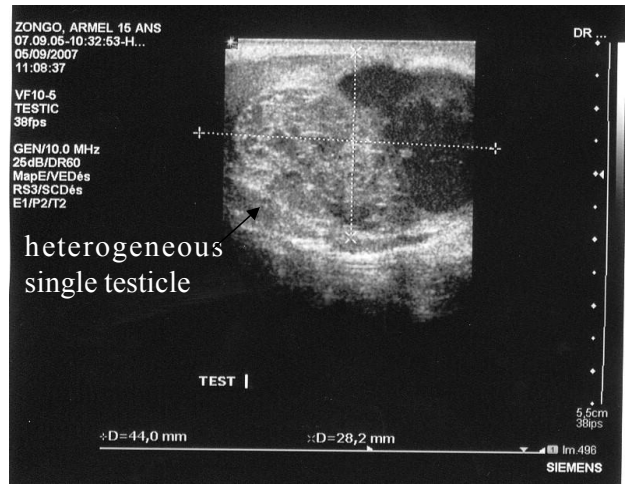

Figure 3. Scrotal ultrasound in axial section showing a heterogeneous single testicle with a dysplastic echopattern. 


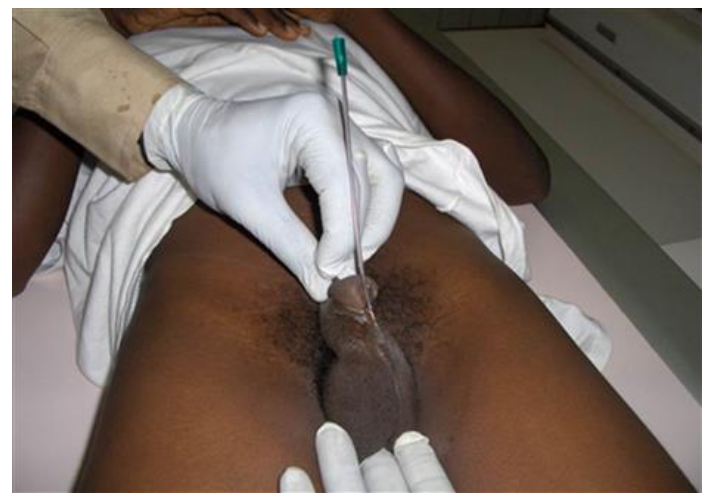

Figure 4. Bladder catheterization through a meatus located below and behind the glans.
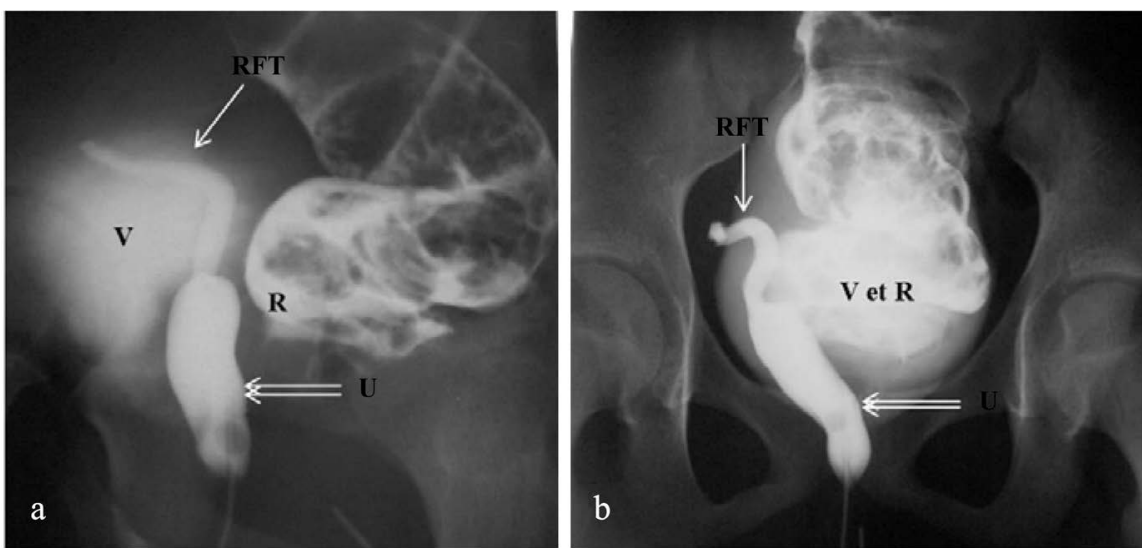

$\mathrm{V}=$ bladder $\mathrm{R}=$ rectum $\mathrm{U}=$ uterus $\mathrm{RFT}=$ right fallopain tube

Figure 5. Genitography: catheterization technique for genitography also showing hypospadias. (a) Oblique view and (b) frontal view showing uterus (double arrows), right fallopian tube (arrow), bladder (V) and rectum (R).

These abnormalities of sexual development remain complex and rare and their management is based on development of an efficient therapeutic strategy [4]. This management requires an accurate diagnosis and involvement of a trained multidisciplinary team [4] [5]. Imaging examinations, including ultrasound and MRI, are considered as key modalities in diagnosis because they provide detailed anatomical information. In our case, ultrasound was the first choice imaging method used to explore the internal genitalia with demonstration of a rudimentary uterus, right ovary and a single testicle.

It is a non-invasive, inexpensive, easily reproducible and relatively accessible technique. Some authors found that it was sensitive and specific in $96 \%$ of cases in morphological screening of internal genitalia [4] [5]. Abdominal ultrasound scan ruled out intra or retroperitoneal pathological mass that might suggest adrenal hyperplasia, a leading cause of ambiguous genitalia. In recent decades, genitography has become more and more important in the diagnosis and therapeutic management of these conditions. It enables excellent viewing of gonads especially when they are small or are not palpable. In our case genitogram gave 
anatomical evaluation and identification of internal genital organs. Thus a small tubular uterus and a large straight smooth right tube, without ampullary folds or peritoneal spillage were demonstrated by contrast medium outlining. Magnetic resonance imaging (MRI) has not been performed for our patient. Some authors revealed that it has better sensitivity (which could reach 100\%) for gonadal exploration compared to ultrasound.

It would be a very useful imaging modality for difficult cases of hermaphroditism, especially in structural analysis of testicular tissues [5]. The choice of sex is of prime importance in care of true hermaphroditism. It must be based on the functional potential of the external genitalia and gonads according to radiological findings. Advancement in imaging modalities, hormone panel, molecular biology and surgical techniques have made a significant contribution to improved diagnosis, which has become more accurate and faster, as well as to the management of children challenging with sexual ambiguity. There is controversy over the decision to choose sex. From the surgical point of view, it is easier to rebuild the external genitalia in order to obtain a female sex. A therapeutic stimulation test with androgens is essential. There are also controversies regarding the age of surgery. The decision is collegial and relies on external genitalia anatomy and reconstruction possibilities, internal genital anatomy and functional abilities.

Sex assignment should be made quickly; surgical treatment must be early and if possible in one time. Diagnosis and treatment must begin at birth to avoid gender identity disturbances. Delay management should consider risk of malignancy and fertility impairment. In our case, the right testicle was dysplastic, thus raising the risk of long-term degeneration. Authors have noted that the incidence of gonad tumors is approximately $10 \%$ in true $46, \mathrm{XY}$ hermaphrodites and $4 \%$ in true hermaphrodites $46, \mathrm{XX}$ [6]. In our context, the cultural beliefs that stigmatize these patients heavily influence the care that is already made difficult by multiple deficiencies. As recommended by Mure and Sirol, sex assignment of children carrying DDS should be done within a multi-disciplinary team including endocrinologists, surgeons, psychologists, cytogeneticists, molecular biologists, hormonal biochemists, obstetricians and urologist [7].

\section{Conclusion}

Genitalia ultrasound scan remains the first-line assessment in investigation of disorders of sexual differentiation in our context. It is most often supplemented by fluoroscopic genitography. Our observation has just reminded us of the good practices of these exams. The accurate anatomical assessment performed by medical imaging, enables important therapeutic options. Sex assignment requires multidisciplinary decision and parental will.

\section{References}

[1] Oilvy-Stuart, A.L. and Brain, C.E. (2004) Early Assessment of Ambiguous Genitalia. 
Archives of Disease in Childhood, 89, 401-407.

https://doi.org/10.1136/adc.2002.011312

[2] Hughes, I.A., Houk, C., Ahmed, S.F. and Lee, P.A. (2006) LWPES Consensus Group ESPE Consensus Group, Consensus statement on Management of Intersex Disorders. Archives of Disease, 91, 554-563. https://doi.org/10.1136/adc.2006.098319

[3] Diakité, M.L., Berthé Ha, J.G., Timbelya, A., Dialloa, M., Maiga, M., Diakité, A., Dialloa, M., Ouattara, K. and Faure, B. (2013) Problem about Care in Disorders of Sexual Differentiation in the Service of Urology: CHU G-Spot. Progress in Urology, 23, 66-72. https://doi.org/10.1016/j.purol.2012.10.008

[4] Goultaiene, A., Elmortaji, K., Sentissi, R., Moataz, A., Rabii, R., Aboutaib, R., Dakir, M., Debbagh, A. and Meziane, F. (2016) Place of the Laparoscopy in Care of Disorders of the Sexual Differenciation: About 4 Cases. Pan African Medical Journal, 23, 167.

[5] Nasir, A.M., et al. (2014) Radiological Imaging in the Patients Affected by 46 XY, Disorders of the Sexual Differenciation (DSD): An Experience of 25 Years in a Major Teaching Hospital. Scholars Journal of Applied Medical Sciences (SJAMS), 2, 2063-2067.

[6] Hadjidekov, G., Kirova, V., Minkov, M. and Vlassov, V. (2007) Contribution of the Medical Imaging in Diagnosis at a 3-Month-Old Infant with True Hermaphroditism. Newspaper of Radiology, 88, 80-83.

[7] Mure, Y., Gelas, T., Ripepi, M., Hoch, M. and Mouriquand, P. (2013) Prise en charge de l'hyperplasie congénitale des surrénales chez la fille. Care in the Congenital Hyperplasia of Suprarenal Glands at the Girl. Progres en Urologie, 13, 1381-1391. 